\title{
Telemedicine Experience for Chronic Care in COPD
}

\author{
Paula de Toledo, Silvia Jiménez, Francisco del Pozo, Senior Member, IEEE, \\ Josep Roca, Albert Alonso, and Carmen Hernandez
}

\begin{abstract}
Information and telecommunication technologies are called to play a major role in the changes that healthcare systems have to face to cope with chronic disease. This paper reports a telemedicine experience for the home care of chronic patients suffering from chronic obstructive pulmonary disease (COPD) and an integrated system designed to carry out this experience. To determine the impact on health, the chronic care telemedicine system was used during one year (2002) with 157 COPD patients in a clinical experiment; endpoints were readmissions and mortality. Patients in the intervention group were followed up at their homes and could contact the care team at any time through the call center. The care team shared a unique electronic chronic patient record (ECPR) accessible through the web-based patient management module or the home visit units. Results suggest that integrated home telemedicine services can support health professionals caring for patients with chronic disease, and improve their health. We have found that simple telemedicine services (ubiquitous access to ECPR, ECPR shared by care team, accessibility to case manager, problem reporting integrated in ECPR) can increase the number of patients that were not readmitted $(51 \%$ intervention, $33 \%$ control), are acceptable to professionals, and involve low installation and exploitation costs. Further research is needed to determine the role of telemonitoring and televisit services for this kind of patients.
\end{abstract}

Index Terms-Chronic care, electronic patient record, home care, telemedicine, telemonitoring.

\section{INTRODUCTION}

$\mathbf{T}$ HE number of people suffering from chronic diseases is rising [1] and so are the costs for their care. These and other facts create significant challenges to the current healthcare systems, which were mainly designed to look after acute episodes, and therefore, are not adapted to the kind of care required by chronic diseases. The management of chronic patients has become a key area of research, and it is increasingly accepted that

Manuscript received February 22, 2005; revised May 10, 2005 and August 27, 2005. This work was supported in part by the European Union under Research Grant CHRONIC (Information Society Technologies, Vth framework programme) and in part by the Spanish Ministry of Health and Consumer Affairs under Research Grant "Red de Telemedicina."

P. de Toledo is with the Informatics Department, Carlos III University, 28903 Madrid, Spain, on leave from the Bioengineering and Telemedicine Research Group (GBT), Universidad Politecnica de Madrid, 28040 Madrid, Spain (e-mail: paula@gbt.tfo.upm.es).

S. Jiménez was with the Bioengineering and Telemedicine Research Group (GBT), Universidad Politecnica de Madrid, 28040 Madrid, Spain. She is now with the University of Alcalá, Madrid, Spain.

F. del Pozo is with the Biomedical Engineering and Telemedicine Research Group, (GBT), Universidad Politecnica de Madrid, 28040 Madrid, Spain.

J. Roca and C. Hernandez are with the Pneumology Service (ICT), Hospital Clinic, IDIBAPS, 08036 Barcelona, Spain, and also with the University of Barcelona, Barcelona, Spain.

A. Alonso is with the Information Systems Department, Hospital Clinic, 08036 Barcelona, Spain, and also with the University of Barcelona, Barcelona, Spain

Digital Object Identifier 10.1109/TITB.2005.863877 changes in the way care is provided are needed to ensure quality and cost containment. Central components of these emerging models are: 1) home-based continuous follow-up of patients; 2) shared care by multidisciplinary teams of professionals from all healthcare levels; 3 ) patient empowerment (adoption of selfmanagement skills); and 4) tailoring of the care processes on a per patient basis. Some authors [2] outline the pivotal role that information and communication systems may have as facilitators of these changes.

We understand that chronic care would benefit from an integrated approach (similar strategies can be found in other reported experiences [3]-[5]) oriented to the following objectives.

1) Provide telemedicine services that support the emerging models for the care for chronic conditions, oriented to patient-centered healthcare, home care, and multidisciplinary teams sharing patient's care.

2) Provide these services in an integrated way taking advantage of the complementarities among them and allowing to tailor the level of services to a particular patient's needs.

3) Achieve a good integration in the existing organizational and technical infrastructure.

4) Grant access to the services using standard Internet protocols for communications and ubiquitous web-based user interfaces applications.

This paper describes the design and implementation of a telemedicine system for the care of chronic patients that follows this approach and a clinical experiment with chronic obstructive pulmonary disease (COPD) patients, using the system.

\section{METHODS}

\section{A. Services Description}

According to the aforementioned objectives, the research team involved in the project identified a set of telemedicine services that might be provided to chronic patients and their carers (Table I).

\section{B. Description of the System}

As no technological platform offering the set of services identified (Table I) was commercially available, the design and implementation of a suitable platform was required. The chronic care telemedicine system developed, shown in Fig. 1, consists of a telemedicine server, located at what we have called the Chronic Care Management Center (CCMC), several user terminals that provide access to the services available, and a network interconnecting all the elements.

1) Telemedicine Server: The telemedicine server consists of a call center, an application server, and an educational material 
TABLE I

SERVices PRovided By THE Chronic CARE PlatForm

\begin{tabular}{|c|c|}
\hline $\begin{array}{l}\text { Services for } \\
\text { professionals }\end{array}$ & $\begin{array}{l}\text { - Electronic Chronic Patient Record (ECPR) : Electronic Patient } \\
\text { Record tailored to the needs of chronic patients' follow-up } \\
\text { (including socio-sanitary information, home visit reports, frailty } \\
\text { report). } \\
\text { - ECPR shared among professionals belonging to different } \\
\text { organizations (hospital, primary care centers). } \\
\text { Ubiquitous access to ECPR, even by home visit teams when } \\
\text { visiting a patient at home. }\end{array}$ \\
\hline $\begin{array}{l}\text { Services for } \\
\text { patients }\end{array}$ & $\begin{array}{l}\text { - Direct access to specialized nurse case manager to report } \\
\text { problems and get solutions and advice. Problem reporting } \\
\text { integrated in ECPR. Possibility of using different access media } \\
\text { (telephone, text messaging and videoconference). } \\
\text { Telemonitoring: remote monitoring of biomedical parameters } \\
\text { (namely one lead ecg, spirometry, pulse-oximetry, blood pressure, } \\
\text { heart rate) and symptoms (questionnaire). Monitoring results } \\
\text { integrated in ECPR. } \\
\text { Televisit (consultation with Case Manager using videoconference) } \\
\text { - Access from home to educational material in a friendly way. }\end{array}$ \\
\hline
\end{tabular}

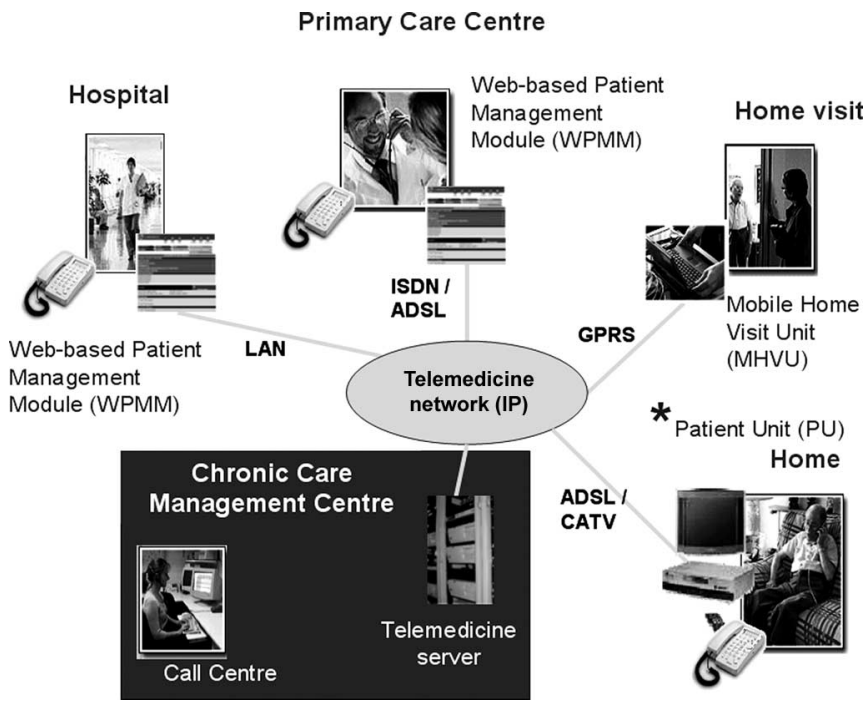

Fig. 1. Chronic Care Telemedicine System (patient unit not used in the trial).

server. Support for videoconference with the patient at the home is also provided.

The call center uses computer-telephony integration (CTI) technology. It consists of a PC with a four-line CTI board (configured as two inbound, two outbound). Phone calls to the system are automatically associated to a patient (through the telco operator caller identification service) and logged into the electronic chronic patient record (ECPR) for follow-up. Depending on the service's needs, phone calls can either be answered by a member of the care team or handled through an automated voice response unit. In both cases a first question is asked to find out if the call is related to health or to administrative problem. If the call is health related, a further questionnaire of ten questions regarding severity of illness is asked.

The application server hosts three different applications: the Web-based Patient Management Module (WPMM), the telemonitoring module, and the home visit server.

The core of the system is the WPMM that gives access to the ECPR. This health record has been tuned to the specific needs of the day-by-day follow-up of the COPD patients and consists of seven sections: work plan, sociosanitary information (frailty report, Fig. 2), treatment, visit's reports (both at home and hospital), referrals, log of phone calls to the call center, and home monitoring results. The WPMM can be accessed by all the members of the care team from any location (patient's home, hospital, primary care center). This piece of software has been developed using application server pages (ASP) and Javascript. It runs on a Microsoft Internet Information Server and uses Open DataBase Connectivity (ODBC) to access a Sybase database management system.

The telemonitoring module is in charge of receiving monitoring data sent both by the patient units (not used in the clinical trial) and the mobile home visit unit (MHVU) (carried to the homes by a visiting nurse), and storing them in the ECPR.

The educational material server is designed to provide education both to patients and professionals, either through web pages or using video-on-demand technology.

All the components of the home telemedicine server are fully integrated as they share a unique ECPR database. Furthermore, an Interapplication Message Management module is added to provide real-time communication among modules when needed. This module dispatches messages triggered by predefined events (arrival of a message or monitoring session result, abnormal results, etc.) to the modules where action on these events is expected, and can be easily configured by the system's manager to adapt the way the system reacts to different events.

The ECPR is integrated with the hospital information system through an HL7 gateway. Information exchanged is a summary of reported monitoring data (from ECPR to HIS) and discharge letters (from HIS to ECPR).

2) Terminals: The laptop-based MHVU is a device to be used by nurses when visiting the patients at home. It provides access to the ECPR (including all the functionality of the WPMM), a calendar tool for visit scheduling, and a spirometer for respiratory monitoring at home. The spirometer (Siebel, Barcelona) is connected through the serial port using the proprietary protocol provided by the manufacturer. Spirometry results and changes to the ECPR are transmitted to the telemedicine server, where they are stored. To make the best of the available bandwidth and achieve acceptable results when used over a cellular GSM/GPRS connection, the system includes a proprietary synchronization protocol that updates the laptop's copy of the patients' database only when needed (and only with information of patients whose visits are planned for each day). This protocol is encapsulated in Secure HyperText Transfer Protocol (HTTPS) for secure transmission. A personal digital assistant (PDA) version for Universal Mobile Telecommunications System (UMTS) networks was designed after the study described here.

The platform developed also included a patient unit [6], not used in the clinical trial, designed to be used by patients at their homes, offering telemedicine services, such as telemonitoring, communication with the case manager using text messages, televisit, and access to educational web material and videos.

3) Network: Telemedicine server and user terminals are connected through the IP-based telemedicine network. For each 


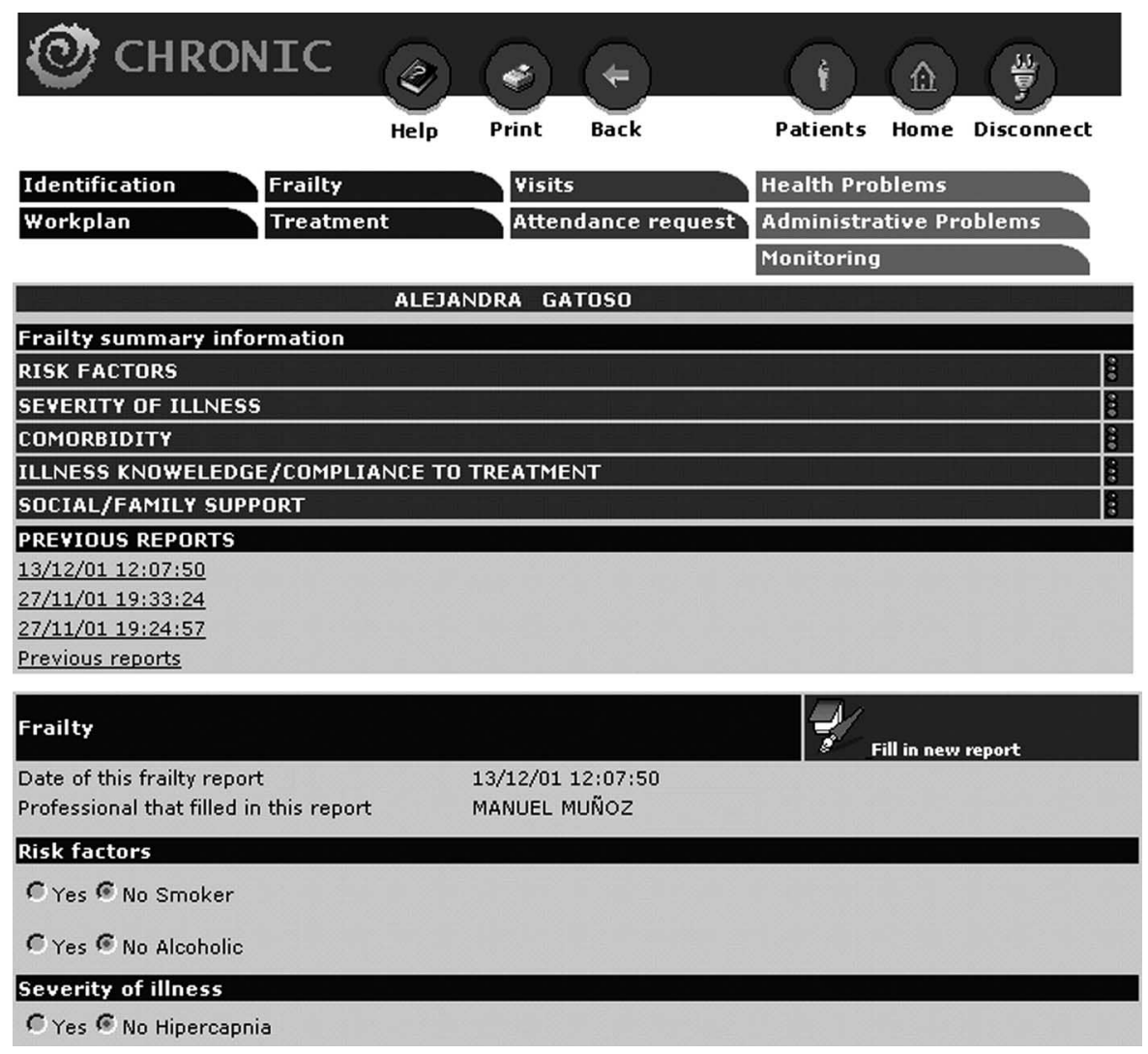

Fig. 2. WPMM. ECPR. Frailty report (partial view). Traffic lights are used as a visual summary of report information.

TABLE II

SECURITY IN THE Chronic CARE TELEMEDICINE System

\begin{tabular}{|c|c|c|c|c|c|c|c|}
\hline & \multirow{2}{*}{$\begin{array}{l}\text { Type } \\
\text { of } \\
\text { access }\end{array}$} & \multicolumn{3}{|c|}{ Authentication } & \multicolumn{3}{|c|}{ Transport security } \\
\hline & & $\begin{array}{l}\text { Login / } \\
\text { passw. }\end{array}$ & $\begin{array}{c}\text { PKI } \\
\text { Certificate }\end{array}$ & Token & $\begin{array}{c}\text { SSL } \\
\text { Encryp } \\
\text { tion }\end{array}$ & VPN & $\begin{array}{l}\text { Intranet } \\
\text { Only }\end{array}$ \\
\hline $\begin{array}{l}\text { Hospital professional's } \\
\text { PC - WPMM server }\end{array}$ & Web & $\mathrm{x}$ & $\mathrm{x}$ & & $\mathrm{x}$ & & $\mathrm{x}$ \\
\hline $\begin{array}{l}\text { Other professional's PC } \\
\text { - WPMM server }\end{array}$ & Web & $\mathrm{x}$ & & $\mathrm{x}$ & $\mathrm{x}$ & $\mathrm{x}$ & \\
\hline $\begin{array}{l}\text { Home visit professional } \\
- \text { MHVU server }\end{array}$ & $\begin{array}{l}\text { Data } \\
\text { (GSM/ } \\
\text { GPRS) }\end{array}$ & $\mathrm{x}$ & & $\mathrm{x}$ & $\mathrm{x}$ & & \\
\hline PU - Monitoring server & Data & & $\mathrm{x}$ & & $\mathrm{x}$ & & \\
\hline $\begin{array}{l}\text { PU - Educational } \\
\text { material server }\end{array}$ & Web & $\mathrm{x}$ & & & $\mathrm{x}$ & & \\
\hline
\end{tabular}

section of the network, the most appropriate telecommunications infrastructure is selected according to the requirements of the final user and the service to be provided, namely bandwidth, availability, mobility, security, and cost. Hospital and primary care centers are connected by a dedicated ISDN line, patients' homes are connected either by a cable TV operator, if they need videoconference service, or GSM otherwise.

Security was a prime concern. Different security needs were identified within the system, according to the user, content of information exchanged, type of access, and location, and they were addressed accordingly (Table II).

\section{Evaluation Methodology}

To evaluate the system in clinical practice, a clinical experiment was designed. The aim of the experiment was to evaluate the impact on COPD patients' health in a hospitalization prevention scenario based on a minimal intervention after an exacerbation episode [7]. As well, we wanted to determine the system's usage patterns, acceptability to professionals, and costs.

The experiment lasted for one year (2002). A total of 157 COPD patients were recruited during a hospital admission due to an acute episode, and randomly distributed in an intervention $\left(n=67\right.$, age $=71 \pm 8$ years; $\mathrm{FEV}_{1}=42 \pm 20 \%$, $2.3 \%$ women $)$ and a control $\left(n=90\right.$, age $=72 \pm 8, \mathrm{FEV}_{1}=$ $42 \pm 15 \%, 3.2 \%$ women) group. [ $\left[\mathrm{FEV}_{1}\right.$ stands for forced expiratory volume in one second].

The experiment was performed from a third level hospital, but involved professionals from primary care also. Care was coordinated by a hospital-based specialized nurse (case manager) and involved a specialist and other hospital professionals (nurses, 43 respiratory lab technicians), three primary care professionals (not specialized in respiratory patients), and two nurses (care manager and assistant) doing home visits. Informed consent was obtained from all patients.

The supporting technology used in this experiment by the patients was limited to the call center. Professionals used the WPMM or the MHVU when visiting the homes. One spirometry test was made at each home visit. Professionals received a short $(2 \mathrm{~h})$ introductory session on the use of the technology. Later 
TABLE III

ACCEPTABILITY TO PROFESSIONALS

\begin{tabular}{lcc}
\hline \hline System acceptability (8 questions) & Mean & SD \\
\hline \hline I am satisfied with the use of the system & 4.25 & 0.50 \\
I feel I provide the right attention to patients using this system & 3.50 & 0.58 \\
I would use the system again & 4.00 & 0.00 \\
I would recommend it to others & 4.00 & 0.00 \\
I find the system easy to use & 4.25 & 0.50 \\
I find the use of the system is stressing (inverted question) & 3.75 & 0.96 \\
The training received is adequate & 3.00 & 1.15 \\
The system improves the care provided to patients & 4.50 & 0.58 \\
\hline \hline
\end{tabular}

(1= strongly disagree, $5=$ strongly agree $)$

they had informal support either from their peers or from the development team.

Patients in the intervention group received an educational session of 1.5-h duration and a single home visit (24-72 $\mathrm{h}$ after discharge), and had telephone access to the system's call center. For these patients, the multidisciplinary team used the system developed to coordinate their work and access the ECPR. Patients in the control group did not have access to the call center, but they received education and home visits as well (number of home visits decided by care team according to the patient evolution and needs). Follow-up for this group was made without the support of WPMM and MHVU.

The call center was manned by a teleoperator during normal working hours and was available both to patients and primary care professionals. Phone calls were transferred as appropriate to care team members. When the operator or case manager was not available, an automated response system collected the call information and stored the query for further reply. Call center is not intended to deal with emergency calls, and the automated response system instructed patients to contact emergency services if needed.

Health-related endpoints measured were number of readmissions, number of visits to Emergency Room, and mortality. Acceptability to professionals was measured using an $a d$ hoc prepared questionnaire (Table III) adapted from Martinez [8]. The questionnaire contains eight items evaluated on a five point Likert scale. Patterns of use were characterized using automatic records made by the different software applications available to the users. Equipment costs for all the hardware and software purchased were registered. Communication costs were calculated from rates actually paid by the hospital and publicly published rates.

\section{Results OF THE ClinicAl EXPERIMENT}

\section{A. Health Outcomes}

As a result of the intervention we found a significant reduction (Table IV) in the number of readmissions in one year, both in number of patients that were not readmitted and in
TABLE IV

HEALTH OUTCOMES

\begin{tabular}{lccc}
\hline \hline & Intervention & Control & $\begin{array}{c}p \text { (level of } \\
\text { significance) }\end{array}$ \\
\hline \hline Patients that were not readmitted (\% patients) & $51.7 \%$ & $33.3 \%$ & 0.04 \\
Patients readmitted at least once & $46.9 \%$ & $65.2 \%$ & 0.03 \\
$\quad$ Patients readmitted more than once & $21.9 \%$ & $29.2 \%$ & 0.35 \\
Number of readmissions per patient & $0.90 \pm 1.28$ & $1.33 \pm 1.73$ & 0.04 \\
Number of visits to Emergency Room & $0.36 \pm 0.98$ & $0.54 \pm 1.12$ & 0.15 \\
Mortality & $20.3 \%$ & $16.9 \%$ & 0.67 \\
\hline \hline
\end{tabular}

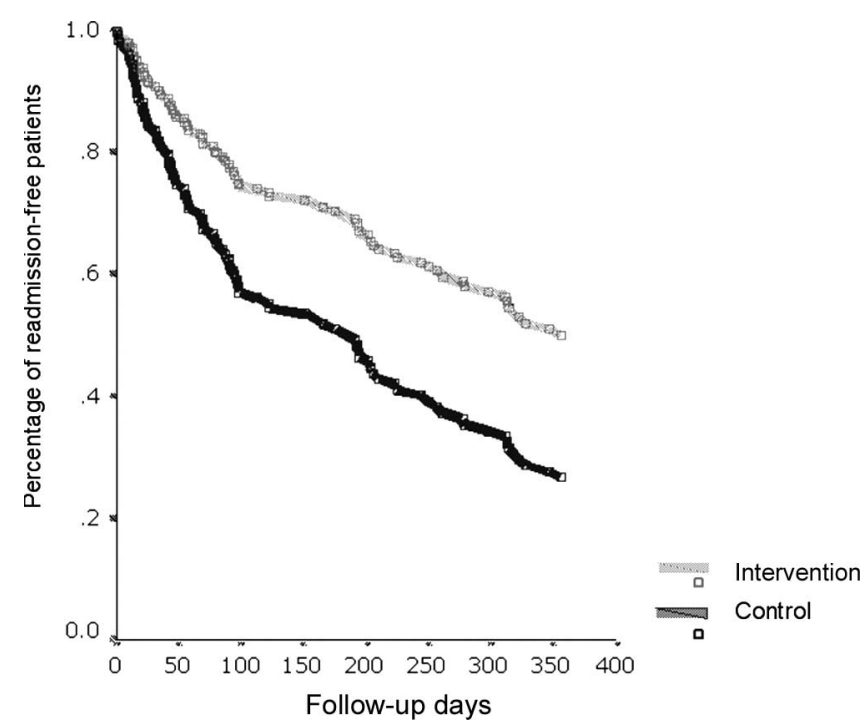

Fig. 3. Evolution of readmission-free patients during the trial.

those readmitted only once. As shown in Fig. 3, patients in the intervention group stayed longer at home before the first readmission. Results are expressed as mean $+\mathrm{SD}$ or as percentages in the corresponding categories. Comparisons between the two study groups were performed using independent $t$-tests, a nonparametric test (Mann-Whitney $U$-test), or the Chi-squared test.

Qualitative comments from the case manager suggest that exacerbations have been prevented due to prompt detection of symptoms and that more treatment changes have been prescribed to patients in the control group.

\section{B. Acceptability to Professionals}

Table III shows the results obtained. The questionnaire was answered by six out of the eight professionals who used the system. Best valued aspects were that it improves patients care and that it is easy to use. The weakest aspect is the training received to use the system, which should be improved.

\section{Characterization of the Patterns of Use of the System}

During one year, 802 sessions took place using the WPMM module and 509 with the home visit unit. The call center received 180 phone calls. 
1) WPMM and Home Visit Unit: Among all the services in the system, the access to the ECPR (view/update record) through the WPMM has been the most frequently used feature. This functionality is similar to that provided by traditional EPR systems, however 1) it enables information sharing among professionals from different care centers and 2) it can be accessed from everywhere, even the patient's home.

The system has been used more frequently by hospital professionals compared to professionals from primary care (6\% of WPMM, 12\% MHVU). As expected, the case manager was the main user of the system (62\% WPMM, 54\% MHVU).

We have not found a significant difference in the access to the different subsets of the ECPR according to the organization (primary care/hospital). Among these data, the most frequently used have been those related to the follow-up (35\%) and treatment $(18 \%)$, and then the frailty report $(7 \%)$ and work plan (7\%).

The mean duration of a session with the WPMM has been $15: 17 \pm 2: 47$ min (range 3:23-17:45), whereas the sessions with the home visit unit were much shorter $(3: 02 \pm 5: 25$, range 1:18-28:04).

The access time with the MHVU to a full page of patients' data (treatment data was used for this test) varied from 5 to $10 \mathrm{~s}$. Access either from hospital or primary care centers was instantaneous.

2) Call Center: Sixty one percent of the patients contacted the call center during the trial. Mean number of phone calls was 16.1 (range $0-90)$. The great majority $(90 \%)$ of the patients' problems notified through the call center was responded within the same day. Health related problems (71\%) abounded more than administrative questions (29\%). Sixty six percent of the phone calls were answered by a teleoperator or the case manager, whereas the automatic system received the rest. Mean duration of a phone call was $1: 54 \pm 2: 04$ min (range $0: 32-11: 50$ ). From the calls that were answered by the automatic system, only $9 \%$ were classified as urgent by the caller.

\section{Costs}

Costs are calculated from equipment cost (hardware and software costs, installation, and maintenance), communications during one year (resulting from system's use figures), personnel, and training. As the experiment was carried out with own staff and this policy is understood to be sustainable for the future, personnel costs are not included. Amortization period considered for equipment is 36 months. Software development costs are not included.

The cost of the clinical experiment was $€ 38932$ ( $€ 36469$ equipment, 1656 communications). Although a proper cost analysis was not carried out, we find it interesting to compare this figure with the cost for 1 day admission in COPD reported for this hospital and patient population [9], which is $€ 220$ per day, showing that the reduction of hospitalization days (Table IV) will pay for the system before the end of the first year (mean duration of hospitalization is 2.8 days, the number of patients considered is 157).

\section{DISCUSSION}

This paper reports an experience on the use of telemedicine in COPD patients' care, based on a technological platform, the chronic care telemedicine system, which supports care coordination and provides ubiquitous access to ECPR and telephone support to patients. The system is scalable for future functionality and provides integration both at information and services level.

The clinical experiment suggests that integrated home telemedicine services and care coordination can support health professionals caring for COPD patients and improve patients' health. Simple services such as ubiquitous access to ECPR, ECPR shared by care team, accessibility to case manager, or problem reporting integrated in ECPR can improve patients' outcomes (remarkable increase in the number of patients that did not need a readmission-51\% intervention, 33\% control). Patients in the program can have a close contact with the care team without requiring frequent visits. The system is acceptable to both professionals and patients, and its installation and exploitation costs are relatively low.

The study indicates that the ready accessibility to the case manager and the availability of all patient information to support decision making result in an intervention that is better tailored to each patient's needs. Both these aspects could be improved without a technological platform as the one described in this work; however, the use of technology offers some important advantages: 1) it supports keeping record of all the interactions with the patients (including phone calls) in an automatic way; as well as tools to follow-up in case demands remain unsolved, 2) it provides ubiquitous access to the up-to-date patient information to the care team, supporting care coordination; and 3) the number of patients that can be provided with closer follow-up is higher, without increasing the workload of the professionals (specialist and case manager). It is likely that the degree of care personalization provided to patients could not have been achieved without the aid of the technology.

A possible weakness of the study is the bias introduced by the physician and/or case manager, as it cannot be organized as double blind (i.e., if information about the patient is available at the WPMM, the physician needs to know this). Although patients were randomized at the start of the study, bias introduced by patients is also possible as they may have experienced a feeling of being better cared-for that may have improved their outcomes. Further research is needed to show that the benefits of a closer relationship between the patient and care team are sustained when the service is provided as a clinical routine.

It is also necessary to determine the number of patients that can be cared for by a single team and to quantify the degree of treatment personalization achieved, for example, by comparing number of treatment changes in control and treatment groups.

Further research is needed as well to determine the type of patients that may benefit from a more complex home intervention supported by the technological platform (telemonitoring and televisit), as we now know that a simple intervention as 
described in this paper is inexpensive and effective with most patients. This finding is consistent with other reports in 1) cardiac rehabilitation in the home [10], suggesting that ECG telemonitoring was only useful to enroll higher risk patients, whereas all others could obtain the same results with a simple telephone connection during the training session and 2) a randomized trial for home telecare in congestive heart failure [11] where the reduction of readmissions was similar for a group with telephone care and other with televisits and telemonitoring. A recently reported elderly telemonitoring program based on a simple question and answer device [12] also supports this view. In COPD, according to our study team's opinion, a telephonic-, internet-, or WAP-based symptoms questionnaire, as also reported by some researchers [13], [14], might be more useful than spirometry and pulse-oximetry telemonitoring, or may complement the information they provide, that is, similar to what is done in the field of diabetes, which is a pioneer in telemedicine for chronic care.

Our experience is an example of a type of service for chronic patients that is being implemented in growing numbers. As compared to other studies, we understand that the contribution of our works lies in the following.

1) The system provides a single picture of all information needed for patient care independently from where it has been gathered (hospital discharge reports, home telemonitoring results, home visits, log of patients' phone calls, case manager follow-up visits...). All this information is important when making decisions on patient care, and should be available. Furthermore, telemonitoring information is fed back to hospital information system avoiding a side-effect of home telemonitoring systems mentioned in a recent study by Salvador et al. [15], the progressive slimming down of the patients' clinical record.

2) The system is oriented to facilitate care coordination among primary care, case manager, and specialized care. All these professionals share the WPMM and use it as a collaborative working tool (schedule specialist or home visits, send messages to other professionals, view visit agenda). Other home telemedicine systems are either directed only to primary care and case managers [12], integrate specialists in a marginal way (faxing occasional reports) [3], or are addressed only to specialists [4], [15]. Care coordination is understood to have a crucial role in chronic patient care and allows adapting the level of care received by each patient to his/her needs at each moment and a smooth transfer among levels when required (exacerbations).

3) The system provides all the services for home telemedicine in a single and integrated platform. Initial home telemedicine experiences were based on a single service (i.e., telemonitoring, telephone follow-up, or televisit). Later research evolved towards telemedicine programs providing a combination of services, tailored to the specific needs of each patient group and healthcare organization [13]-[16]. This trend started with diabetes [4]. Our platform provides a technical infrastructure that can support simultaneously telemedicine programs for differ- ent chronic diseases and patients with different risk levels. As an example of this, the hospital where the clinical trial took place plans to use the platform in the near future for experiences with diabetes and congestive heart failure.

Platforms like the Chronic Care Telemedicine System are called to play a major role as facilitators of the new strategies for chronic care management, through out-of-hospital follow-up, patient empowerment, and care coordination, improving patient care without an associated cost raise. To achieve this goal, it is crucial to redefine the roles of the professionals involved, being, from our findings, a case manager and a technical/logistic support office two key functions. A teleoperator would only be necessary in programs with high numbers of patients, whereas in smaller programs like this one the phone call workload can be easily handled by the case manager.

It is worth mentioning the role that the widespread availability of Internet and Web browsers has played in the advance shown by home telemedicine in the past five years. The main technological barrier remaining to the deployment of integrated home telemedicine systems is the high cost of telemonitoring devices.

\section{ACKNOWLEDGMENT}

The authors want to acknowledge assistance of the patients and their families, as well as staff involved in the experiment, especially J. A. Montero.

\section{REFERENCES}

[1] J. Epping-Jordan, Innovative Care for Chronic Conditions, Geneva: World Health Organization, 2001.

[2] E. H. Wagner, C. Davis, J. Schaefer, M. Von Korff, and B. Austin, "A survey of leading chronic disease management programs: Are they consistent with the literature?" Manag. Care Q, vol. 7, no. 3, pp. 56-66, 1999.

[3] J. Finkelstein and R. H. Friedman, "Telemedicine system to support asthma self-management," in Proc. IEEE EMBS Int. Conf. Inform. Technol. Appl. Biomed., 2000, pp. 164-167.

[4] E. J. Gómez et al., "Telemedicine as a tool for intensive management of diabetes: The DIABTel experience," Comput. Methods Programs Biomed., vol. 69, pp. 163-177, Aug. 2002.

[5] S. Shea et al., "Columbia university's informatics for diabetes education and telemedicine (IDEATel) project: Rationale and design," J. Amer. Med. Inform. Assoc., vol. 9, no. 1, pp. 49-62, Jan.-Feb. 2002.

[6] P. De Toledo, S. Jimenez, and F. Del Pozo, "A telemedicine system to support a new model for care of chronically ill patients," J. Telemed. Telecare, vol. 8, pp. 17-19, 2002.

[7] B. Hernández et al., "Efectos de una intervención mínima en la prevención de hospitalizaciones de pacientes con EPOC," Arch. Bronconeumol., vol. 39, suppl. 2, p. 44, Jun. 2003.

[8] A. Martínez, R. Rodrigues, A. Infante, C. Campillo, and C. Gattini, Bases Metodológicas Para Evaluar la Viabilidad y el Impacto de Proyectos de Telemedicina, Washington: Organización panamericana de la salud, 2001.

[9] C. Hernandez et al., "Home hospitalization of exacerbated COPD patients. A randomized controlled trial of clinical efficacy and costs of services for chronically ill patients," Eur. Respir. J., vol. 21, no. 1, pp. 58-67, Jan. 2003.

[10] P. A. Ades, F. J. Pashkow, G. Fletcher, I. L. Pina, L. R. Zohman, and J. R. Nestor, "A controlled trial of cardiac rehabilitation in the home setting using electrocardiographic and voice transtelephonic monitoring," Amer. Heart J., vol. 139, no. 3, pp. 543-548, Mar. 2000.

[11] A. F. Jerant, R. Azari, and T. S. Nesbitt, "Reducing the cost of frequent hospital admissions for congestive heart failure: A randomized trial of a home telecare intervention," Med. Care, vol. 39, no. 11, pp. 1234-1245, Nov. 2001.

[12] R. S. Schofield et al., "Early outcomes of a care coordination-enhanced telehome care program for elderly veterans with chronic heart failure," Telemed J E Health, vol. 11, no. 1, pp. 20-27, Feb. 2005. 
[13] J. Finkelstein, M. R. Cabrera, and G. Hripcsak, "Internet-based home asthma telemonitoring: Can patients handle the technology?" Chest, vol. 117, no. 1, pp. 148-155, Jan. 2000.

[14] M. Young, D. Sparrow, D. Gottlieb, A. Selim, and R. Friedman, "A telephone-linked computer system for COPD care," Chest, vol. 119, no. 5, pp. 1565-1575, May 2001.

[15] C. H. Salvador et al., "Airmed-cardio: A GSM and Internet services-based system for out-of-hospital follow-up of cardiac patients," IEEE Trans. Inf. Technol. Biomed., vol. 9, no. 1, pp. 73-85, Mar. 2005.

[16] C. Caceres et al., "A home integral telecare system for HIV/AIDS patients," Stud. Health Technol. Inform., vol. 114, pp. 23-29, 2005.

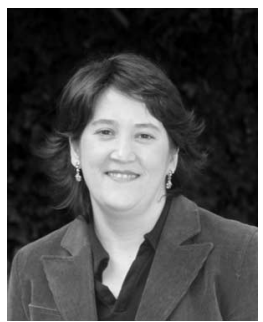

Paula de Toledo received the M.S. and Ph.D. degrees from the Universidad Politecnica de Madrid (UPM), Madrid, Spain, in 1995 and 2003, respectively, both in telecommunication engineering.

In 1996, she joined the Biomedical Engineering and Telemedicine Group (GBT), UPM, where she was a full-time Research Fellow and participated in more than ten EU-funded projects, and contracts with industrial companies in the field of telemedicine and home health care. She is currently a Visiting Professor at the Carlos III University, Madrid, Spain. Her current research interests are integrated telemedicine systems for the care of chronic patients, sensor networks and ambient intelligence for telemedicine applications, health care service modeling, and intelligent alarm generation in telemedicine applications.

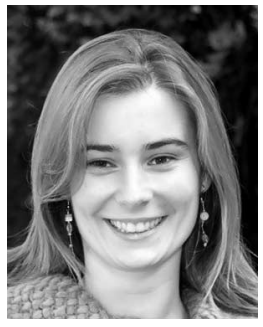

Silvia Jiménez received the M.S. degree in telecommunication engineering from the Universidad Politecnica de Madrid (UPM), Madrid, Spain, in 2000.

Since 1999, she has been with the Biomedical Engineering and Telemedicine Group (GBT), UPM, where she has participated in many EU and Nationalfunded research projects related to Home Health Care and Telemedicine. Currently, she is an Associate Professor at the Universidad de Alcala, Madrid, Spain. Her main interests are health states characterization based on automatic classification of long-term physiological data, and ambient intelligence and sensor networks for health care purposes.

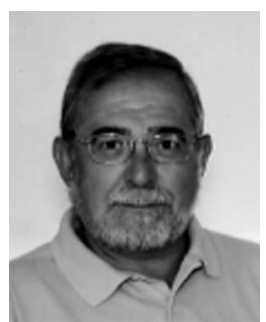

Francisco del Pozo (SM'04) received the Telecomm. Eng. degree and the Ph.D. degree in telecommunication engineering from the Universidad Politécnica of Madrid (UPM), Madrid, Spain, in 1969 and 1979, respectively.

In 1985, he joined the Biomedical Engineering and Telemedicine Group (GBT), UPM, as the Founder and Director, where in 1986, he became a Full Professor. He has been a Research Fellow at the Physics Department, Imperial College, London; Neurobiology Department, University of Yale; Department of Laboratory Medicine and Pathology, University of Minnesota; and the Biophysics and Physiology Department, University of New York. He has been an Invited Professor at several U.S. and EU universities. He has been a Scientific Advisor of main medical and telecommunication companies and health care institutions. He has directed more than 350 scientific papers and $20 \mathrm{Ph} . \mathrm{D}$. dissertations. He is the Main Researcher of more than 40 EU-funded research projects and 28 contracts with industrial companies.

Dr. Pozo is the Founder and President of the Sociedad Española de Telemedicina (SET); and Vice-president of the Sociedad Española de Ingeniería Biomédica. He is the Founder and Chief Editor of International Telemedicine and a Board Member of the Journal of Telemedicine and Telecare.

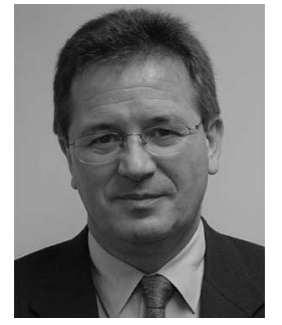

Josep Roca received the M.D. and Ph.D. degrees from the University of Barcelona, Barcelona, Spain, in 1975 and 1983, respectively.

$\mathrm{He}$ is with the Pneumology Service, Hospital Clinic, Barcelona, Spain, where he is the Director of the Pulmonary Physiology Unit. He is also a Professor of Medicine at the University of Barcelona. $\mathrm{He}$ is the author of 160 original articles published in peer-reviewed journals ( $>85 \%$ first quartile, impact factor 875) editor of three books, and author of 51 book chapters and 50 solicited review papers. His main area of interest has traditionally been pulmonary gas exchange and exercise. Currently, he is devoted to research on innovative models of care for chronic patients using information and communication technologies as enabling tools.

Dr. Roca has been a member of the Committee on Health Care and Quality of Life within the Strategic Plan for the IST of the Generalitat de Catalunya and currently he is the Chairman of the Committee on Chronic Care of the Generalitat de Catalunya. He has executive responsibilities in medical and scientific societies. During 2000-2002, he was the President of the European Respiratory Society (ERS)

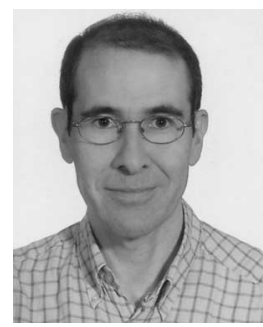

Albert Alonso was born in Barcelona, Spain, in 1962. He received the M.D. and Ph.D. degrees from Barcelona University, Barcelona, in 1986 and 1992 respectively, and the postgraduate degree in management and organization of information systems from the Universitat Pompeu Fabra, Barcelona, in 1996.

$\mathrm{He}$ is currently a Senior Researcher responsible for the Technological Innovation Unit at the Information Systems Department, Hospital Clinic, Barcelona. He has an extensive experience in European R\&D projects. He is also a Lecturer in Medical Informatics, University of Barcelona, and co-responsible for one of the modules of the International Master Course on Health Information Management (Erasmus University, Rotterdam, Holland). His main research area focuses on definition and evaluation of emerging models in healthcare provisions, in particular, those based on the continuum of care across providers and how to couple information technology to enhance health service provisions.

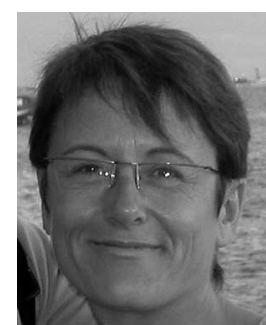

Carmen Hernandez was born in Barcelona, Spain, in 1961. She received the degree in nursing from the University of Barcelona, Barcelona, in 1982.

Currently, she is with the Pneumology Service, Hospital Clinic, Barcelona, Spain, where she is the Coordinator of Integrated Care Programs in COPD, and teaches continuum education for nursing postgraduates at the University of Barcelona. Her current research activity is focused on the evaluation of chronic patients and implementation of the Integrated Care program for fragile chronic patients, to improve resources and reduce costs.

Ms. Hernandez is a member of the Scientific Committee of the Nursing Group in the American Thoracic Society (ATS) and the Chair of the Nursing Group in the European Respiratory Society (ERS). 